Homology, Homotopy and Applications, vol. 15(2), 2013, pp.53-71

\title{
HOMOLOGICAL DIMENSIONS OF RING SPECTRA
}

\author{
MARK HOVEY AND KEIR LOCKRIDGE
}

(communicated by J. Daniel Christensen)

\begin{abstract}
We define homological dimensions for $S$-algebras, the generalized rings that arise in algebraic topology. We compute the homological dimensions of a number of examples, and establish some basic properties. The most difficult computation is the global dimension of real $K$-theory $K O$ and its connective version $k o$ at the prime 2 . We show that the global dimension of $K O$ is 2 or 3 , and the global dimension of $k o$ is 4 or 5 .
\end{abstract}

\section{Introduction}

The authors have been engaged in trying to develop homological dimensions for the ring objects that arise in algebraic topology. These are cohomology theories with some kind of cup product that is associative up to infinitely coherent homotopy. These are commonly called $S$-algebras, among other names, and a standard reference is [EKMM97].

Such $S$-algebras are analogous to rings, but they have no elements, and they do not have abelian module categories. What they do have is a triangulated derived category that generalizes the derived category of an ordinary associative ring $R$. Indeed, in this case, there is an Eilenberg-MacLane spectrum $S$-algebra $H R$, and $\mathcal{D}(H R)$ is naturally equivalent to $\mathcal{D}(R)$ as a triangulated category.

Thus, to develop the ring theory of $S$-algebras $E$, we need to work with their derived categories $\mathcal{D}(E)$. In previous papers $[\mathbf{H L 0 9 b}, \mathbf{H L 1 1}]$, the authors have developed definitions of right global dimension r. gl. dim. $E$ and ghost dimension gh. dim. $E$ for $S$-algebras $E$ and proved that these generalize the usual notions of right global dimension and weak dimension for rings. We have also studied $S$-algebras for which these dimensions are 0 .

The object of this paper is to study the homological dimensions of the $S$-algebras that arise in nature. After an initial section in which we recall the basics of our theory of global dimension and ghost dimension of $S$-algebras, we discuss examples in Section 2. The sphere, $M U$, and $B P$ all have infinite global and ghost dimensions, whereas $E_{n}$ has ghost and global dimension $n$. For a commutative $S$-algebra $E$ such

Received May 7, 2011, revised May 30, 2012; published on August 20, 2013.

2010 Mathematics Subject Classification: 55P43, 16E10, 18E30.

Key words and phrases: ring spectrum, global dimension.

Article available at http://intlpress.com/HHA/v15/n2/a3 and doi:10.4310/HHA.2013.v15.n2.a3

Copyright (C) 2013, International Press. Permission to copy for private use granted. 
that $E_{*}$ is Noetherian and has finite global dimension, such as $E_{n}$ or $K$, we have

$$
\text { gl. } \operatorname{dim} . E=\text { gh. } \operatorname{dim} . E=\text { gl. } \operatorname{dim} . E_{*} \text {. }
$$

This fails when gl. dim. $E_{*}=\infty$, however. We show that, at the prime 2 , gl. dim. $K O$ is either 2 or 3 , and gl. dim. $k o$ is either 4 or 5 , whereas both rings $K O_{*}$ and $k o_{*}$ have infinite global dimension. We also show that gl. dim. tmf is finite at the prime 3 .

In Section 3 we prove some basic properties of global and ghost dimension. The most important point is that these dimensions are not Morita invariant. In fact, it is already true that the global and weak dimensions of ordinary rings are not invariant under derived equivalences, and we give an example communicated to us by Lidia Angeleri Hügel. We have been unable to generalize many of the basic properties of the global dimension of Noetherian rings, however. This might be because we have no intrinsic definition of $E$ being a Noetherian $S$-algebra; we just assume $E_{*}$ is right Noetherian. So, for example, we do not know whether r. gl. dim. $E=$ gh. dim. $E$ when $E_{*}$ is right Noetherian. We end the paper with a brief section on $S$-algebras $E$ with global dimension 1 . We had originally thought this would mean $E_{*}$ would have to be 1Gorenstein, in analogy with the fact that gl. $\operatorname{dim} . E=0$ implies $E_{*}$ is quasi-Frobenius, and in fact this is true, but only with additional assumptions on $E_{*}$.

\section{Acknowledgments}

The authors would like to thank Lidia Angeleri Hügel for the example mentioned above, Ben Wieland for pushing us to consider the global dimension of $K O$, and Stefan Schwede for the example that proves the global dimension of $K O$ is at least 2.

\section{Homological dimensions}

The object of this section is to define our various homological dimensions of ring spectra and to prove basic relations between them. Recall that $E$ is an $S$-algebra, and $\mathcal{D}(E)$ denotes the derived category of right $E$-modules. This is a compactly generated triangulated category, and when $E$ is the Eilenberg-MacLane spectrum $H R$ of an ordinary ring $R$, then $\mathcal{D}(E)$ is equivalent to the usual unbounded derived category of $R$.

We begin by defining projective, injective, and flat objects of $\mathcal{D}(E)$.

Definition 1.1. An object $X \in \mathcal{D}(E)$ is said to be projective (resp. injective, resp. flat) if $X_{*}$ is a projective (resp. injective, resp. flat) $E_{*}$-module. A map $f: X \rightarrow Y$ in $\mathcal{D}(E)$ is said to be ghost if $f_{*}=0$, and $f$ is said to be phantom if $\mathcal{D}(E)(A, f)=0$ for all compact $A \in \mathcal{D}(E)$.

The basic properties of these objects and maps are summed up in the following proposition.

Proposition 1.2. Suppose $E$ is an S-algebra.

1. If $M$ is a projective or injective $E_{*}$-module, then there is an $X \in \mathcal{D}(E)$ (necessarily projective or injective) with $X_{*} \cong M$. 
2. If $M$ is a flat $E_{*}$-module, and $\mathcal{D}(E)$ is a Brown category (see [HPS97, Section 4.1]), for example if $E_{*}$ is countable, then there is an $X \in \mathcal{D}(E)$ (necessarily flat) such that $X_{*} \cong M$.

3. $X$ is a projective object of $\mathcal{D}(E)$ if and only if the natural map

$$
\mathcal{D}(E)(X, Y) \rightarrow \operatorname{Hom}_{E_{*}}\left(X_{*}, Y_{*}\right)
$$

is an isomorphism for all $Y \in \mathcal{D}(E)$. This is true if and only if every ghost with domain $X$ is null.

4. $X$ is an injective object of $\mathcal{D}(E)$ if and only if the natural map

$$
\mathcal{D}(E)(Y, X) \rightarrow \operatorname{Hom}_{E_{*}}\left(Y_{*}, X_{*}\right)
$$

is an isomorphism for all $Y \in \mathcal{D}(E)$. This is true if and only if every ghost with codomain $X$ is null.

5. $X$ is a flat object of $\mathcal{D}(E)$ if and only the natural map

$$
X_{*} \otimes_{E_{*}} Y_{*} \rightarrow \pi_{*}\left(X \wedge_{E} Y\right)
$$

is an isomorphism for all left E-modules $Y$. This is true if and only if every ghost with domain $X$ is phantom.

Proof. The first part is well-known. In fact, it is proved in [BKS04, Proposition A.4] that every $E_{*}$-module of projective or injective dimension $\leqslant 2$ is realizable. We prove part (3) next. If $X$ is projective, then the universal coefficient spectral sequence of [EKMM97, Theorem IV.4.1] implies that

$$
\mathcal{D}(E)(X, Y) \cong \operatorname{Hom}_{E_{*}}\left(X_{*}, Y_{*}\right) .
$$

This in turn implies that there are no nontrivial ghosts with domain $X$. Now, if there are no nontrivial ghosts with domain $X$, construct a projective module $P_{*}$ and an epimorphism $P_{*} \rightarrow X_{*}$. This is then realizable, as we have just seen, by a map $P \rightarrow X$, whose cofiber $X \rightarrow Y$ is a ghost. This map is thus null, so $X$ is a retract of $P$, and hence projective. The proof of part (4) is similar. Part (5) is proved in [HL11].

Turning to part (2), suppose $M$ is a flat $E_{*}$-module. Then $M_{*}$ is a directed colimit of finitely generated projective $E_{*}$-modules $Q_{i}$. We can then realize this system by compact projective $E$-modules $P_{i}$, with $\pi_{*} P_{i} \cong Q_{i}$, by part (3). If $\mathcal{D}(E)$ is a Brown category, we can then take the minimal weak colimit [HPS97, Section 4.2] of the diagram of the $P_{i}$ to obtain an $X$ with $X_{*} \cong \operatorname{colim} Q_{i} \cong M$.

Once we have projective, injective, and flat objects, we should then be able to define the projective dimension of an arbitrary object. This is a little more complicated than in the abelian setting, but has in fact already been worked out by Christensen in [Chr98]. We note that what we call projective dimension is not the dimension with respect to the projective class determined by the projectives above, as one would find in relative homological algebra and in [Chr98], but instead is one less than the length determined by that projective class. This is unfortunate terminology on our part, but this choice was made in $[\mathbf{H L O 9 b}]$ and so is difficult to change now. We also think that triangulated categories are fundamentally different from abelian categories, and so the abelian theory of dimension should be modified for the triangulated setting. 
Definition 1.3. Let $E$ be an $S$-algebra, and $X$ an object of $\mathcal{D}(E)$. We define the projective dimension (resp. constructible flat dimension) of $X$, written proj. dim. $X$ (resp. con. flat dim. $X$ ), inductively as follows. We define proj. dim. $X=$ 0 (resp. con. flat $\operatorname{dim} . X=0$ ) if and only if $X$ is projective (resp. flat). Then we define proj. $\operatorname{dim} . X \leqslant n+1$ (resp. con. flat $\operatorname{dim} . X \leqslant n+1$ ) if and only if there is an exact triangle

$$
Y \rightarrow P \rightarrow \tilde{X} \rightarrow \Sigma Y
$$

where $P$ is projective (resp. flat), proj. $\operatorname{dim} . Y \leqslant n$ (resp. con. flat $\operatorname{dim} . X \leqslant n$ ), and $X$ is a retract of $\tilde{X}$. We define the flat dimension of $X$, written flat $\operatorname{dim}$. $X$, to be the smallest integer $n$ for which any composite of $n+1$ ghosts with domain $X$ is phantom, or $\infty$ if there is no such $n$. The reader may well wonder why we have two notions of flat dimension. The answer is that we have been unable to prove they are equivalent, and the constructible flat dimension is the more obvious one, but the flat dimension is the more useful one. See Proposition 1.4 below and [HL11] for details.

We can define injective dimension similarly to the way we defined projective dimension, but it would be more usual to define the injective dimension of $X$, written inj. $\operatorname{dim} . X$, inductively as follows. We define inj. $\operatorname{dim} . X=0$ if and only if $X$ is injective, and inj. $\operatorname{dim} . X \leqslant n+1$ if and only if there is an exact triangle

$$
\Sigma^{-1} Y \rightarrow \tilde{X} \rightarrow I \rightarrow Y
$$

where $I$ is injective, inj. $\operatorname{dim} . Y \leqslant n$, and $X$ is a retract of $\tilde{X}$.

The major difference between this definition and the definition of the analogous dimensions in abelian categories is the fact that $X$ itself need not appear in an exact triangle with things of smaller dimension, but must only be a retract of such a thing. Without this condition, Proposition 1.4 below would be false.

Proposition 1.4. Let $E$ be an $S$-algebra, and let $X$ be an object of $\mathcal{D}(E)$.

1. proj. dim. $X \leqslant n$ if and only if every composite of $n+1$ ghosts

$$
f_{n+1} \circ f_{n} \circ \cdots \circ f_{1}
$$

is null, where the domain of $f_{1}$ is $X$. This is true if and only if in the universal coefficient spectral sequence

$$
E_{2}^{s, t}=\operatorname{Ext}_{E_{*}}^{s, t}\left(X_{*}, Y_{*}\right) \Rightarrow \mathcal{D}(E)(X, Y)_{t-s}
$$

we have $E_{\infty}^{s, *}=0$ for all $s>n$ and all objects $Y$ of $\mathcal{D}(E)$.

2. inj. $\operatorname{dim} . X \leqslant n$ if and only if every composite of $n+1$ ghosts $f_{n+1} \circ f_{n} \circ \cdots \circ f_{1}$ is null, where the codomain of $f_{n+1}$ is $X$. This is true if and only if in the universal coefficient spectral sequence

$$
E_{2}^{s, t}=\operatorname{Ext}_{E_{*}}^{s, t}\left(Y_{*}, X_{*}\right) \Rightarrow \mathcal{D}(E)(Y, X)_{t-s}
$$

we have $E_{\infty}^{s, *}=0$ for all $s>n$ and all objects $Y$ of $\mathcal{D}(E)$.

3. flat $\operatorname{dim} . X \leqslant$ con. flat $\operatorname{dim} . X$, and flat $\operatorname{dim} . X \leqslant n$ if and only if the following equivalent properties hold: 
(a) In the universal coefficient spectral sequence

$$
E_{s, t}^{2}=\operatorname{Tor}_{s, t}^{E_{*}}\left(X_{*}, Y_{*}\right) \Rightarrow \pi_{t-s}\left(X \wedge_{E} Y\right)
$$

we have $E_{s, *}^{\infty}=0$ for all $s>n$ and all objects $Y$ of $\mathcal{D}\left(E^{\mathrm{op}}\right)$.

(b) There is an exact triangle

$$
A \rightarrow X \stackrel{g}{\rightarrow} W \rightarrow \Sigma A
$$

in which proj. $\operatorname{dim} . A \leqslant n$ and $g$ is phantom.

(c) Every map $F \rightarrow X$ from a compact E-module $F$ factors through a compact $B$ with proj. dim. $B \leqslant n$.

Proof. For part (1), the first statement is contained in [Chr98, Theorem 3.5]. The second statement follows from the construction and naturality of the universal coefficient spectral sequence [EKMM97, Section IV.5]. Indeed, suppose $E_{\infty}^{s, *}=0$ for all $s>n$ and all $Y$, and suppose

$$
X \stackrel{f_{1}}{\longrightarrow} X_{1} \stackrel{f_{2}}{\longrightarrow} \cdots \stackrel{f_{n+1}}{\longrightarrow} X_{n+1}
$$

is a composite of $n+1$ ghosts. Each of the maps $f_{i}: X_{i} \rightarrow X_{i+1}$ has positive filtration. Therefore, their composition will have filtration $\geqslant n+1$ by Proposition IV.4.4 of [EKMM97], and therefore will be null. For the converse, note that in the construction of the universal coefficient spectral sequence, we construct exact triangles

$$
\begin{gathered}
\Sigma^{-1} X_{1} \rightarrow P_{0} \stackrel{g_{0}}{\longrightarrow} X \stackrel{h_{1}}{\longrightarrow} X_{1} \\
\Sigma^{-1} X_{2} \rightarrow P_{1} \stackrel{g_{1}}{\longrightarrow} X_{1} \stackrel{h_{2}}{\longrightarrow} X_{2} \\
\vdots \\
\Sigma^{-1} X_{j+1} \rightarrow P_{j} \stackrel{g_{j}}{\longrightarrow} X_{j} \stackrel{h_{j+1}}{\longrightarrow} X_{j+1}
\end{gathered}
$$

where $P_{j}$ is projective and $\pi_{*} g_{j}$ is onto for all $j$, and so $h_{j+1}$ is a ghost for all $j$. A map has filtration $n+1$ if and only if it factors through the composite

$$
X \stackrel{h_{1}}{\longrightarrow} X_{1} \stackrel{h_{2}}{\longrightarrow} \cdots \stackrel{h_{n+1}}{\longrightarrow} X_{n+1} .
$$

Thus every map of filtration $n+1$ is a composite of $n+1$ ghosts. Therefore, if proj. dim. $X \leqslant n$, then every map of filtration $n+1$ is null.

Part (2) is then completely dual. Part (3) is proved in [HL11].

The following fact is also useful.

Proposition 1.5. Let $E$ be an $S$-algebra, and let $X$ be an object of $\mathcal{D}(E)$. Then

$$
\text { proj. } \operatorname{dim} . X \leqslant \text { proj. dim. } E_{*} X_{*}, \quad \text { inj. dim. } X \leqslant \operatorname{inj.~dim.~} E_{*} X_{*},
$$

and

flat $\operatorname{dim} . X \leqslant$ con. flat $\operatorname{dim} . X \leqslant$ flat $\operatorname{dim} . E_{*} X_{*}$.

The first two statements of this proposition are obvious given Proposition 1.4, and the last statement is proved in [HL11].

We can now define our homological dimensions. 
Definition 1.6. Suppose $E$ is an $S$-algebra. Define the (right) global dimension of $E$, r. gl. dim. $E$, by

$$
\text { r. gl. } \operatorname{dim} . E=\sup _{X} \text { proj. } \operatorname{dim} . X=\sup _{X} \operatorname{inj} . \operatorname{dim} . X .
$$

These two numbers are equal because they are both equal to the longest nontrivial composition of ghosts in $\mathcal{D}(E)$ (or $\infty$ if there are arbitrarily long nontrivial compositions of ghosts). In case $E$ is a commutative $S$-algebra, we just refer to the global dimension, gl. dim. $E$. Define the ghost dimension of $E$, gh. dim. $E$, by

$$
\text { gh. } \operatorname{dim} . E=\sup _{X \text { compact }} \text { proj. } \operatorname{dim} . X=\sup _{X} \text { flat } \operatorname{dim} . X .
$$

It is proven in [HL11] that the two definitions of ghost dimension given above coincide.

We then have the following theorem that sums up the basic properties of these definitions.

Theorem 1.7. Suppose $E$ is an $S$-algebra.

1. gh. dim. $E \leqslant$ r. gl. dim. $E$.

2. r. gl. dim. $E \leqslant$ r.gl. dim. $E_{*}$, with equality if $E=H R$ for an ordinary ring $R$.

3. gh. $\operatorname{dim} . E \leqslant$ w. $\operatorname{dim} . E_{*}$, with equality if $E=H R$ for an ordinary ring $R$.

4. gh. $\operatorname{dim} . E=$ gh. $\operatorname{dim} . E^{\mathrm{op}}$, where $E^{\mathrm{op}}$ is $E$ with the opposite multiplication.

The first part of this theorem is obvious. Part (2) is proved in [HL09a], though it is originally in the second author's thesis. Parts (3) and (4) are proved in [HL11].

$S$-algebras of global dimension 0, studied in [HL09b], are called semisimple. In particular, there are semisimple ring spectra with r. gl. dim. $E_{*}=\infty$. Similarly, $S$-algebras of ghost dimension 0 are called von Neumann regular, and are also studied in [HL09b].

\section{Examples}

We would, of course, like to compute gh. $\operatorname{dim} . E$ and r.gl. dim. $E$ for various $S$ algebras $E$. Some of this was done in $[\mathbf{H L O 9 b}$, where the authors classified the semisimple $S$-algebras $E$ if either $E_{*}$ is commutative or local. Those spectra are rather unusual, however. We address some of the more common $S$-algebras $E$ in this section, concentrating on the case when $E_{*}$ is Noetherian.

We begin with the sphere spectrum, and the following unsurprising result.

Proposition 2.1. Let $S$ be the sphere $S$-algebra. Then gh. $\operatorname{dim} . S=\operatorname{gl} . \operatorname{dim} . S=\infty$.

Proof. This is due to Christensen [Chr98], who provides bounds on proj. $\operatorname{dim} . \mathbb{R} P^{n}$ (which he calls the length). In particular, a lower bound for proj. $\operatorname{dim} . \mathbb{R} P^{k}$ is given by the longest nonzero chain of Steenrod operations in its homology, since Steenrod operations are obviously ghosts. And this longest chain is easily seen to grow without bound as $k$ grows.

Corollary 2.2. Let $S_{(p)}$ be the p-local sphere $S$-algebra, where $p$ is an integer prime. Then gh. dim. $S_{(p)}=$ gl. dim. $S_{(p)}=\infty$. 
Proof. Use reduced power operations in the cohomology of the skeleta of $B \mathbb{Z} / p$ to replace Steenrod operations in $\mathbb{R} P^{k}$. We do not need to know the exact length of a nontrivial composition of these operations, we just need to know that this length grows without bound as we take larger skeleta.

Recall that the length of the longest regular sequence in a ring is often called the depth.

Theorem 2.3. If $E$ is a commutative $S$-algebra, then

$$
\operatorname{depth} E_{*} \leqslant \text { gh. } \operatorname{dim} . E \leqslant \min \left\{\text { w. } \operatorname{dim} . E_{*}, \text { r. gl. dim. } E\right\} \leqslant \text { r. gl. dim. } E_{*} .
$$

Proof. In view of Theorem 1.7, it suffices to prove the first inequality. Let

$$
x_{1}, x_{2}, \ldots, x_{n}
$$

be a regular sequence in $E_{*}$. The main algebraic input we need is the computation that

$$
\operatorname{Ext}_{E_{*}}^{i}\left(E_{*} /\left(x_{1}, \ldots, x_{n}\right), E_{*}\right)=0 \text { if } i \neq n
$$

and is nonzero if $i=n$. One can prove this by induction on $n$ (or by the Koszul resolution), using the exact sequences

$$
0 \rightarrow E_{*} /\left(x_{1}, \ldots, x_{k-1}\right) \stackrel{x_{k}}{\longrightarrow} E_{*} /\left(x_{1}, \ldots, x_{k-1}\right) \rightarrow E_{*} /\left(x_{1}, \ldots, x_{k}\right) \rightarrow 0,
$$

where we have ignored suspensions for simplicity.

We also need the fact that there is a $E$-module $E /\left(x_{1}, \ldots, x_{n}\right)$ realizing the $E_{*^{-}}$ module $E_{*} /\left(x_{1}, \ldots, x_{n}\right)$. One can also construct these by induction, using the exact triangles

$$
E /\left(x_{1}, \ldots, x_{i-1}\right) \stackrel{x_{i}}{\rightarrow} E /\left(x_{1}, \ldots, x_{i-1}\right) \rightarrow E /\left(x_{1}, \ldots, x_{i}\right) \rightarrow E /\left(x_{1}, \ldots, x_{i-1}\right),
$$

ignoring suspensions again.

The universal coefficient spectral sequence

$$
\operatorname{Ext}_{E_{*}}^{s, t}\left(E_{*} /\left(x_{1}, \ldots, x_{n}\right), E_{*}\right) \Rightarrow \mathcal{D}(E)\left(E /\left(x_{1}, \ldots, x_{n}\right), E\right)
$$

then has only one non-vanishing line, where $s=n$. It therefore collapses, and so there is an element in $E_{\infty}$ of filtration $n$. Thus gh. $\operatorname{dim} . E \geqslant n$, as required.

It is tempting to believe that Theorem 2.3 works in the noncommutative case as well, as long as we take regular sequences in the center $Z\left(E_{*}\right)$. The algebraic calculation works fine, but we do not seem to be able to construct the necessary maps $x: M \rightarrow M$ for an $E$-module $M$ and an $x \in Z\left(E_{*}\right)$. We can construct such maps for $E$-bimodule maps $x: E \rightarrow E$, but an element in the center of $E_{*}$ need not give such a map, so far as we know.

Corollary 2.4. We have

$$
\text { gh. } \operatorname{dim} . M U=\text { gh. } \operatorname{dim} . B P=\infty,
$$

while gh. dim. $E_{n}=$ gl. dim. $E_{n}=n$ and gh. dim. $K=$ gl. dim. $K=1$. 
Here $E_{n}$ denotes Morava $E$-theory, with

$$
E_{n *} \cong W \mathbb{F}_{p^{n}}\left[\left[u_{1}, \ldots, u_{n-1}\right]\right]\left[u, u^{-1}\right] .
$$

This is known to be a commutative $S$-algebra by [GH04]. The coefficient ring has global dimension $n$, and $p, u_{1}, \ldots, u_{n-1}$ is a regular sequence, so Theorem 2.3 gives the desired result.

This corollary suggests the following more general theorem.

Theorem 2.5. Suppose $E$ is a commutative $S$-algebra such that $E_{*}$ is Noetherian with gl. dim. $\pi_{*} E<\infty$. Then gh. dim. $E=$ gl. dim. $E=$ gl. dim. $E_{*}$.

Proof. The point is that $\operatorname{depth} R=\operatorname{gl}$. $\operatorname{dim} . R$ when $R$ is Noetherian commutative of finite global dimension, so the result follows from Theorem 2.3. This algebraic fact is a corollary of Serre's characterization of regular local rings, but we cite [BH84, Section 2] because it contains an interesting non-commutative generalization as well.

We suspect that Theorem 2.5 may be true even if $E$ is not a commutative $S$ algebra. Much less is known about noncommutative Noetherian rings of finite global dimension, however.

We now address the global dimension of real $K$-theory. Unfortunately, we have not been able to determine the exact value of gl. $\operatorname{dim} . K O$, but we do at least bound it.

Theorem 2.6. Let $K O$ denote 2 -local periodic real $K$-theory and ko denote 2-local connective real $K$-theory, both of which are commutative $S$-algebras. Then

$$
2 \leqslant \text { gl. } \operatorname{dim} . K O \leqslant 3
$$

and

$$
4 \leqslant \text { gl. dim. } k o \leqslant 5 .
$$

Proof. This depends on the results of Bousfield [Bou90] and Wolbert [Wol98]. Bousfield wrote his paper using naive $K O$-module spectra, as opposed to objects in $\mathcal{D}(K O)$. However, Wolbert explains why Bousfield's results hold in $\mathcal{D}(K O)$ as well, and proves analogous results for $\mathcal{D}(k o)$. We note that there is an error in Wolbert's paper [Sag08], but this error is about the realizability of $k o_{*}$ and $K O_{*}$-modules and does not affect this theorem.

Bousfield constructs an abelian category of CRT-modules, which are modules over the 3-object additive category consisting of $\left\{K O_{*}, K_{*}, K S C_{*}\right\}$ and the various standard maps between them. Here $K S C=K O \wedge C\left(\eta^{2}\right)$ is self-conjugate $K$ theory. There is a functor from $K O$-module spectra to this category that takes $X$ to $\pi_{*}^{C R T}(X)$, which is the set $\left\{\pi_{*} X, \pi_{*}(C(\eta) \wedge X), \pi_{*}\left(C\left(\eta^{2}\right) \wedge X\right)\right\}$ together with the maps between them. He then proves that $\pi_{*}^{C R T}(X)$ has projective dimension $\leqslant 1$ for every $K O$-module spectrum $X$. The $K O$-modules $P$ such that $\pi_{*}^{C R T}$ is projective as a CRT-module are coproducts of suspensions of $K O, K$, and $K S C$, and every projective CRT-module arises this way. Furthermore, maps between projective CRTmodules are realizable as maps of the corresponding $K O$-modules. This means that 
for every $K O$-module spectrum $X$, there is a cofiber sequence

$$
Q \rightarrow P \stackrel{f}{\rightarrow} X \stackrel{g}{\rightarrow} \Sigma Q
$$

in $\mathcal{D}(K O)$ in which $Q$ and $P$ are coproducts of suspensions of copies of $K O, K$, and $K S C$. Since $K=K O \wedge C(\eta)$ and $K S C=K O \wedge C\left(\eta^{2}\right)$, we see that any 2-fold composite of ghosts out of $P$ or $Q$ is trivial. Suppose now that $s_{4} \circ s_{3} \circ s_{2} \circ s_{1}$ is a 4 -fold composite of ghosts out of $X$. Then $s_{2} \circ s_{1} \circ f$ is a 2-fold composite of ghosts out of $P$, so is trivial. Therefore, there is a map $h$ with domain $\Sigma Q$ such that

$$
h \circ g=s_{2} \circ s_{1} .
$$

But then

$$
s_{4} \circ s_{3} \circ s_{2} \circ s_{1}=s_{4} \circ\left(s_{3} \circ h\right) \circ g
$$

is the composition of a 2-fold ghost out of $\Sigma Q$ with $g$, so is trivial. Thus the global dimension of $K O$ is at most 3 .

To see that gl. $\operatorname{dim} . K O \geqslant 2$, we use an example shown us by Stefan Schwede. Consider the cofiber sequence of $\mathrm{KO}$-module spectra

$$
K O \vee \Sigma^{2} K O \vee \Sigma^{4} K O \vee \Sigma^{6} K O \rightarrow K U \stackrel{f}{\rightarrow} X
$$

where the first map is determined by the homotopy elements $1, v_{1}, v_{1}^{2}$ and $v_{1}^{3}$. This map is easily seen to be onto on homotopy, so that $f$ is a ghost. We also consider the map

$$
Y \stackrel{g}{\rightarrow} K U \stackrel{i}{\rightarrow} L_{0} K U
$$

where $i$ is the canonical map (of strict ring spectra, so also of $K O$-modules) from $K U$ to its rationalization. Since $K U$ is torsion-free, this map is injective on homotopy, so $g$ is also a ghost. The composite

$$
f \circ g: Y \rightarrow X
$$

is therefore a 2-fold composite of ghosts, and we claim that it is nontrivial. Indeed, if it were trivial, then there would be a map $h: L_{0} K U \rightarrow X$ such that $h i=f$. Thus $f$ is in the image of the map of abelian groups

$$
\mathcal{D}(K O)\left(L_{0} K U, X\right) \rightarrow \mathcal{D}(K O)(K U, X) .
$$

However, the first group is a rational vector space (because $L_{0} K U$ is a rational spectrum), and the second group is a finitely generated abelian group (this follows from a thick subcategory argument using the fact that $K U$ and $X$ are both in the thick subcategory generated by $K O$ ). There are no nonzero maps from a rational vector space to a finitely generated abelian group, so we conclude that $f$ is trivial. This means that $K U$ is a projective $K O$-module, which is obviously false. Thus $g \circ f$ must be nontrivial.

The argument for $k o$ is a little more complicated. Wolbert [Wol98] describes the connective version of CRT-modules that he calls crt-modules, and shows that if $X$ is a $k o$-module, then $\pi_{*}^{c r t}(X)$ has projective dimension at most 2 in the category of 
crt-modules. Wolbert then gives a version of the universal coefficient spectral sequence

$$
E_{2}^{s, t}=\operatorname{Ext}_{c r t}^{s, t}\left(\pi_{*}^{c r t}(X), \pi_{*}^{c r t}(Y)\right) \Rightarrow \mathcal{D}(k o)(X, Y)_{t-s} .
$$

As before, any 2-fold composite of ghosts has filtration 1 in this spectral sequence (since $k$ and $k s c$ are 2-cell complexes in $\mathcal{D}(k o)$ ). Therefore, any 6-fold composite of ghosts will have filtration 3 , but the spectral sequence is trivial above filtration 2 . Thus every 6 -fold composite of ghosts is trivial, so gl. dim. $k o \leqslant 5$.

To see that gh. dim. $k o \geqslant 4$, we use the fact that $k o \wedge A(1)=H \mathbb{F}_{2}$, where $A(1)$ is the usual 8-cell complex with whose cohomology is $\mathcal{A}\langle x\rangle /\left(\mathrm{Sq}^{2^{n}} x \mid n \geqslant 2\right)$. Using this, we can compute $\mathcal{D}(k o)\left(H \mathbb{F}_{2}, H \mathbb{F}_{2}\right)$. It is the subring of the Steenrod algebra generated by $\mathrm{Sq}^{1}$ and $\mathrm{Sq}^{2}$. Since every element of the Steenrod algebra is a ghost, the nontrivial element $\mathrm{Sq}^{2} \mathrm{Sq}^{1} \mathrm{Sq}^{2} \mathrm{Sq}^{1}$ is a nontrivial composite of 4 ghosts, and is a self-map of the compact object $H \mathbb{F}_{2}$ in $\mathcal{D}(k o)$. Thus gh. dim. $k o \geqslant 4$.

The proof of this theorem is of course dependent on knowing an awful lot about $K O$ and $k o$. We have much less information about higher analogues of $K O$, such as the spectrum tmf of topological modular forms [Hop02]. However, it is often the case with such spectra $E$ that there is a finite type 0 spectrum $X$ such that $E \wedge X$ is a Noetherian $S$-algebra of finite global dimension. For example, $K O \wedge C(\eta)=K U$, and at the prime 3 there is a 3-cell complex $T$ such that $\operatorname{tmf} \wedge T$ is a wedge of two copies of $B P\langle 2\rangle$ [Beh06, Lemma 2, after Corollary 2.4.6]. Presumably a larger such finite complex also exists at the prime 2 for $\operatorname{tmf}$, though such a result has not been proven as yet.

In general, given spectra $X$ and $Y$, we can define the term " $Y$ can be built from $X$ in $\ell$ steps" in the same way that we defined the projective dimension. That is, we say that $Y$ can be built from $X$ in 0 steps if $Y$ is a retract of a coproduct of suspensions of $X$. We then say that $Y$ can be built from $X$ in $\ell$ steps if there is an exact triangle

$$
Z \rightarrow W \rightarrow \widetilde{Y} \rightarrow \Sigma Z
$$

where $W$ can be built from $X$ in 0 steps, $Z$ can be built from $X$ in $\ell-1$ steps, and $Y$ is a retract of $\tilde{Y}$.

Theorem 2.7. Suppose $E$ is an S-algebra and $X$ is a spectrum with the following properties.

1. $E \wedge X$ is an E-algebra, so also an $S$-algebra, with r.gl. $\operatorname{dim} .(E \wedge X)=m<\infty$.

2. As an object of $\mathcal{D}(S)$, proj. $\operatorname{dim} . X=k$.

3. $S$ can be built from $X$ in $\ell$ steps.

Then gl. dim. $E \leqslant(k+1)(\ell+1)(m+1)-1$.

Note that if $Y$ is in the thick subcategory generated by $X$, then $Y$ can be built from $X$ in a finite number of steps. In particular, if $X$ is a type 0 finite spectrum, then $S$ can be built from $X$ in a finite number of steps. Hence we get the following corollary.

Corollary 2.8. Suppose $E$ is an $S$-algebra and $X$ is a type 0 finite spectrum such that $E \wedge X$ is an E-algebra with finite right global dimension as an $S$-algebra. Then $E$ has finite right global dimension. 
Here is the proof of Theorem 2.7.

Proof. Note that if $M$ is an $E$-module, then $M \wedge X \cong M \wedge_{E}(E \wedge X)$ is an $E \wedge X$ module. Similarly, if $f$ is a map of $E$-modules, then $f \wedge 1_{X}$ is a map of $E \wedge X$-modules.

Now, let $M$ and $N$ be $E$-modules. We will prove by induction on $t$ that if $f: M \rightarrow$ $N$ is a $(t+1)$-fold ghost and $Z$ has projective dimension $t$, then

$$
f \wedge 1_{Z}: M \wedge Z \rightarrow N \wedge Z
$$

is a ghost map of $E$-modules. Indeed, if $t=0$, then $Z$ is a retract of a coproduct of copies of $E$, so $f \wedge 1_{Z}$ is a retract of a coproduct of copies of $f$. Thus $f \wedge 1_{Z}$ is a ghost when $f$ is. Now suppose that the statement is true for $t=n, f$ is an $(n+2)$-fold ghost, and $Z$ has projective dimension $n+1$. Then $Z$ is a retract of a spectrum $\widetilde{Z}$ such that there is a cofiber sequence

$$
Y \rightarrow P \rightarrow \widetilde{Z} \rightarrow \Sigma Y
$$

where $Y$ has projective dimension $n$ and $P$ is projective. It suffices to show that $f \wedge 1_{\widetilde{Z}}$ is a ghost; for notational clarity we rewrite $\widetilde{Z}$ as $Z$. Write $f=g \circ h$ where $h: M \rightarrow M^{\prime}$ is an $(n+1)$-fold ghost and $g: M^{\prime} \rightarrow N$ is a ghost. Then $h \wedge 1_{\Sigma Y}$ is a ghost. Therefore the map $\pi_{*}\left(h \wedge 1_{Z}\right)$ factors though some map $r: \pi_{*}(M \wedge Z) \rightarrow \pi_{*}\left(M^{\prime} \wedge P\right)$. Hence $\pi_{*}\left(f \wedge 1_{Z}\right)$ factors as a composite

$$
\pi_{*}(M \wedge Z) \stackrel{r}{\rightarrow} \pi_{*}\left(M^{\prime} \wedge P\right) \stackrel{\pi_{*}\left(g \wedge 1_{P}\right)}{\longrightarrow} \pi_{*}(N \wedge P) \rightarrow \pi_{*}(N \wedge Z) .
$$

Since $g \wedge 1_{P}$ is a ghost, this map is zero, and the induction step is proved.

Returning to the statement of Theorem 2.7, we see that if $f$ is a $(k+1)$-fold ghost, then $f \wedge 1_{X}$ is a ghost, necessarily as a map of $E \wedge X$-modules. Hence, if $f: M \rightarrow N$ is a composite of $(k+1)(m+1)$ ghosts, then $f \wedge 1_{X}$ is an $(m+1)$-fold ghost, and hence is null as a map of $E \wedge X$-modules, and in particular as a map of $E$-modules. But then we can proceed by induction on $\ell$ to see that if $Y$ can be built from $X$ in $\ell$ steps, then any $(k+1)(m+1)(\ell+1)$-fold ghost $f$ has $f \wedge 1_{Y}$ null as a map of $E$-modules. Taking $Y=S$ completes the proof.

Corollary 2.9. At the prime 3 , the spectrum tmf of topological modular forms has finite global dimension.

Proof. As mentioned above, there is a 3 -cell complex $T$ such that

$$
t m f \wedge T \simeq B P\langle 2\rangle \wedge \Sigma^{8} B P\langle 2\rangle
$$

by [Beh06]. The complex $T$ has cells in dimensions 0,4 , and 8 , so is obviously type 0 . The spectrum $\operatorname{tmf} \wedge T$ is also called $\operatorname{tm} f_{0}(2)$, and is a commutative tmf-algebra (it is the connective cover of $T M F_{0}(2)$, which is the spectrum of sections over a certain stack of a sheaf of commutative $S$-algebras; see [Beh06]). The homotopy ring of $t m f_{0}(2)$ is polynomial over $\mathbb{Z}_{3}$ (if we use the completed version) on two generators [Hil07, Proposition 2.3], and therefore $\operatorname{tmf}_{0}(2)$ has global dimension 3 by Theorem 2.5. 


\section{Properties}

In this section, we examine some general properties of global and ghost dimension. Most of these properties concern the relationship between the global dimension or ghost dimension of an $S$-algebra $E$ and some other $S$-algebra $F$ related to it. We discuss the cases when $F$ is a smashing Bousfield localization of $E$, when $F$ is a free $E$-module, and when $F$ is Morita equivalent to $E$. We end with a discussion of the relationship between ghost and global dimension. We find this particularly unsatisfactory, however, because we are unable to prove anything along the lines of the well-known algebraic fact that if $R$ is Noetherian or right perfect (which is equivalent to flats being projective), then r.gl. $\operatorname{dim} . R=$ w. $\operatorname{dim} . R$.

Proposition 3.1. Suppose $L$ is a smashing Bousfield localization functor, and $E$ is an $S$-algebra. Then

$$
\text { r.gl. } \operatorname{dim} . E \geqslant \text { r.gl. } \operatorname{dim} . L E \text {. }
$$

We do not know if this theorem is true if the localization functor is not smashing.

Proof. Note first that $L E$ is again an $S$-algebra [EKMM97, Chapter VIII]. The main point is that because $L$ is smashing, the category $L \mathcal{D}(E)$ of $L$-local $E$-modules is equivalent to the category $\mathcal{D}(L E)$ [EKMM97, Proposition VIII.3.2]. Thus a nontrivial composite of ghosts in $\mathcal{D}(L E)$ is the same thing as a nontrivial composite of ghosts between $L$-local objects in $\mathcal{D}(E)$. Hence r. gl. dim. $E \geqslant$ r. gl. dim. $L E$.

The same thing is true for ghost dimension, though we need to assume $\mathcal{D}(E)$ is a Brown category. Recall from Section 4.2 of [HPS97] that this means homology theories, and morphisms between them, are representable.

Proposition 3.2. Suppose $L$ is a smashing Bousfield localization functor, and $E$ is an $S$-algebra such that $\mathcal{D}(E)$ is a Brown category. Then

$$
\text { gh. } \operatorname{dim} . E \geqslant \text { gh. } \operatorname{dim} . L E .
$$

Proof. Because $\mathcal{D}(E)$ is a Brown category, every object of $\mathcal{D}(E)$ is the minimal weak colimit of the compact objects mapping to it, by [HPS97, Theorem 4.2.4]. But then we can follow the proof of $[\mathbf{H S 9 9}$, Theorem $6.2(\mathrm{~b}, \mathrm{c})]$ to show that the compact objects of $L \mathcal{D}(E)$ are the retracts of objects of the form $L F$, for $F$ compact in $\mathcal{D}(E)$. Thus, if we have a nontrivial composite of ghosts

$$
X_{0} \rightarrow X_{1} \rightarrow \cdots \rightarrow X_{n}
$$

in $L \mathcal{D}(E)$, where $X_{0}$ is compact in $L \mathcal{D}(E)$, we can write $X_{0}$ as a retract of $L F$ for some compact object $F \in \mathcal{D}(E)$. Then the composite

$$
F \rightarrow L F \rightarrow X_{0} \rightarrow X_{1}
$$

must be nontrivial, and gives us a nontrivial composite of ghosts out of a compact object in $\mathcal{D}(E)$.

In general, there is not much relationship between the global dimension of an $S$-algebra $E$ and a general $E$-algebra $F$. At one extreme we have the smashing localizations discussed above. The other extreme is $E$-algebras that are free over $E$. 
Proposition 3.3. Suppose $E \rightarrow F$ is a map of $S$-algebras such that $F_{*}$ is free over $E_{*}$. Then r.gl. dim. $E \leqslant$ r. gl. dim. $F$ and gh. dim. $E \leqslant$ gh. dim. $F$.

Note that the inequalities in this proposition can certainly be strict, as we can see by looking at the inclusion of ordinary rings from $\mathbb{Z}$ to $\mathbb{Z}[x]$, for example. We think this proposition should hold even if $F_{*}$ is only assumed to be faithfully flat over $E_{*}$, but have not been able to prove it.

Proof. Consider the extension functor $F \wedge_{E}(-): \mathcal{D}(E) \rightarrow \mathcal{D}(F)$ and its right adjoint, the restriction functor. Because $F_{*}$ is flat over $E_{*}$, the natural map

$$
F_{*} \otimes_{E_{*}} X_{*} \rightarrow \pi_{*}\left(F \wedge_{E} X\right)
$$

is an isomorphism. Indeed, both sides are homology functors on $\mathcal{D}(E)$, and the given map is an isomorphism for $X=E$, so it is an isomorphism for all $X \in \mathcal{D}(E)$. This means that $F \wedge_{E}(-)$ preserves ghosts.

In particular, if $g$ is a composition of $n$ ghosts, and $F_{*}$ is flat over $E_{*}$, then $F \wedge_{E} g$ is a composition of $n$ ghosts. If the domain of $g$ is a compact object of $\mathcal{D}(E)$, then the domain of $F \wedge_{E} g$ is a compact object of $\mathcal{D}(F)$. However, $F \wedge_{E} g$ may be zero, even if $g$ is nonzero. This cannot happen if $F_{*}$ is free over $E_{*}$, however, for then $F \wedge_{E} X$ is a coproduct of copies of $X$ as an $E$-module. Thus $g$ is a retract of the restriction of $F \wedge_{E} g$, so if $g$ is nontrivial, so is $F \wedge_{E} g$.

We now discuss Morita equivalence. Recall from the work of Schwede and Shipley [Sch04] that two $S$-algebras $E$ and $F$ are called Morita equivalent if there is a chain of Quillen equivalences from the model category of $E$-modules to the model category of $F$-modules. Schwede and Shipley prove that, if $E$ and $F$ are Morita equivalent and cofibrant in the model structure on $S$-algebras (we can always assume this, since weak equivalences of $S$-algebras induce Quillen equivalences of the module categories), then there is an $E$ - $F$-bimodule $M$ and an $F$-E-bimodule $N$, both of which are compact both as $E$ and $F$-modules, so that the functors

$$
\Phi(X)=X \wedge_{E} M \quad \text { and } \quad \Psi(Y)=Y \wedge_{F} N
$$

are inverse equivalences, with $\Phi: \mathcal{D}(E) \rightarrow \mathcal{D}(F)$ and $\Psi$ going back the other way. Furthermore, $M$ generates $\mathcal{D}(F)$ and $N$ generates $\mathcal{D}(E)$, in the sense that the smallest localizing subcategory containing $M($ resp. $N)$ is $\mathcal{D}(E)($ resp. $\mathcal{D}(F)$ ).

This is analogous to the usual Morita situation with ordinary rings, with one important difference. The generators $M$ and $N$ do not have to be projective. This means that neither $\Phi$ nor $\Psi$ need preserve projective objects, so that we do not expect global dimension or ghost dimension to be Morita invariant.

Indeed, we thank Lidia Angeleri Hügel for the following example coming from the theory of tilting modules, a reference for which is [ASS06]. Recall that, for an ordinary ring $A$, a tilting module is a module $T$ whose projective resolution gives an equivalence of categories from the derived category of $A$ to the derived category of $\operatorname{End}_{A}(T)$ by using the derived tensor product. In particular, a tilting module defines a Morita equivalence in the above sense between the Eilenberg-MacLane $S$-algebras $H A$ and $H B$. Now, let $A$ be the path algebra of the quiver $1 \rightarrow 2 \rightarrow 3$, so that $A$ is isomorphic to the ring of lower triangular $3 \times 3$ matrices. There are then three 
projective indecomposable $A$-modules $P_{1}, P_{2}$, and $P_{3}$, corresponding to the quiver representations

$$
k \stackrel{\equiv}{\rightarrow} \stackrel{\equiv}{\rightarrow} k, \quad 0 \rightarrow k \stackrel{\rightrightarrows}{\rightarrow} k, \quad \text { and } \quad 0 \rightarrow 0 \rightarrow k .
$$

There are also three dual injective indecomposables $I_{1}, I_{2}, I_{3}=P_{1}$ corresponding to the representations

$$
k \rightarrow 0 \rightarrow 0, \quad k \stackrel{\equiv}{\rightarrow} k \rightarrow 0, \quad \text { and } \quad k \stackrel{\equiv}{\rightarrow} k \stackrel{\equiv}{\rightarrow} k .
$$

We claim that $T=P_{3} \oplus I_{1} \oplus P_{1}$ is a tilting module. Indeed, like any path algebra, $A$ has global dimension $\leqslant 1$ (and in fact r. gl. $\operatorname{dim} . A=1$ ), so proj. $\operatorname{dim} . T \leqslant 1$. We also need $\operatorname{Ext}^{1}(T, T)=0$, and this boils down to $\operatorname{Ext}^{1}\left(I_{1}, P_{3}\right)=0$, which is straightforward. The last condition for $T$ to be a tilting module is for there to be an exact sequence

$$
0 \rightarrow A \rightarrow T_{1} \rightarrow T_{2} \rightarrow 0
$$

where $T_{1}$ and $T_{2}$ are finite direct sums of direct summands of $T$, so finite direct sums of $P_{3}, I_{1}$, and $P_{1}$. But $A$ corresponds to the representation

$$
k \rightarrow k \oplus k \rightarrow k \oplus k \oplus k
$$

where the maps are inclusions of the obvious summands. Thus we can just take $T_{2}=I_{1}$ and $T_{1}=P_{3} \oplus P_{1} \oplus P_{1}$ with the obvious surjection from $T_{1}$ to $T_{2}$.

So we have a Morita equivalence between $H A$ and $H B$, where

$$
B=\operatorname{End}_{A}(T) .
$$

One can compute $B$ directly. It is a 5 -dimensional algebra generated by the orthogonal idempotents $e_{1}, e_{2}, e_{3}$ and two other elements $\alpha, \beta$, where the only nonzero products involving $\alpha$ and $\beta$ are

$$
e_{3} \alpha=\alpha e_{1}=\alpha \quad \text { and } \quad e_{2} \beta=\beta e_{3}=\beta .
$$

Let $M$ be the right $B$-module which has dimension 1 over $k$, where $e_{2}$ acts by the identity and the other generators of $B$ act trivially. Then one can check that proj. $\operatorname{dim} . M=2$, so

$$
\text { r. gl. } \operatorname{dim} . B \geqslant 2>\text { r. gl. dim. } A=1 \text {. }
$$

In fact, r. gl. $\operatorname{dim} . B=2$. Note that both $A$ and $B$ are Noetherian, so their weak dimensions are also different. Thus $H A$ and $H B$ have different ghost dimensions as well.

We should mention that the property of having global dimension 0 is Morita invariant, at least if one of the two $S$-algebras has commutative homotopy [HL09b, Proposition 2.11]. We now prove that the finiteness of global dimension is at least Morita invariant.

Proposition 3.4. Suppose $E$ and $F$ are Morita equivalent $S$-algebras. Then $E$ has finite right global dimension (resp. finite ghost dimension) if and only if $F$ does so.

For example, this means that the endomorphism $S$-algebra of any finite type 0 spectrum has infinite global dimension. In general, we expect r. gl. dim. $E$ to be always infinite if $E$ is a finite spectrum. 
Proof. As mentioned above, we can assume that $E$ and $F$ are cofibrant $S$-algebras, and we have compact generators $M$ of $\mathcal{D}(F)$ and $N$ of $\mathcal{D}(E)$ that are also bimodules, so that smashing with $M$ and $N$ give inverse equivalences. Now suppose $f$ is a ghost map in $\mathcal{D}(E)$. Then $f \wedge_{E} M$ has the property that $\mathcal{D}(F)\left(M, f \wedge_{E} M\right)_{*}=0$. Since $M$ is a compact generator of $\mathcal{D}(F), F$ is in the thick subcategory generated by $M$. Thus, $F$ can be built from $M$ in finitely many steps (see the discussion preceding Theorem 2.7). In particular, there is an integer $k$ such that, if $f$ is a composite of $k$ ghosts in $\mathcal{D}(E)$, then $f \wedge_{E} M$ is a ghost in $\mathcal{D}(F)$. In particular, if $F$ has finite global dimension, say $n$, then if $f$ is a composite of $k(n+1)$ ghosts in $\mathcal{D}(E)$, then $f \wedge_{E} M$ is null. Since smashing with $M$ is an equivalence of categories, this means that $f$ is null, so $E$ has finite global dimension.

Reversing the roles of $E$ and $F$ gives the reverse implication. For the ghost dimension, we repeat the same argument using a compact object as the source of our first ghost map. This causes no problems since the equivalences of categories preserve compact objects.

We now look for a relationship between the global dimension and the ghost dimension. In algebra, we have the well-known inequality

$$
\text { r. gl. } \operatorname{dim} . R \leqslant \text { w. } \operatorname{dim} . R+\text { pure gl. } \operatorname{dim} . R,
$$

where pure gl. $\operatorname{dim} . R$ is the pure global dimension of $R$. This can actually be replaced by the maximum projective dimension of a flat module. Indeed, in a projective resolution of an arbitrary $R$-module $M$, the syzygies $M_{k}$ are flat whenever $k \geqslant \mathrm{w}$. $\operatorname{dim}$. $R$. But this means our projective resolution is also a projective resolution of the flat module $M_{\mathrm{w} . \operatorname{dim} . R}$, giving us the desired inequality.

One might expect the analogue of the pure global dimension to be the phantom dimension, defined below.

Definition 3.5. Suppose $E$ is an $S$-algebra. Define the phantom dimension of $E$, phan. dim. $E$, to be the smallest integer $n$ such that every composite of $n+1$ phantom maps in $\mathcal{D}(E)$ is zero, or $\infty$ if there exist arbitrarily long such nonzero composites.

Recall that a phantom map is a map $f$ for which $[C, f]_{*}=0$ for every compact $E$-module $C$. So, if we think of a map whose cofiber is a ghost as the homotopytheoretic analogue of an epimorphism, then a map whose cofiber is a phantom is the homotopy-theoretic analogue of a pure epimorphism, and the phantom dimension should be analogous to the pure global dimension. Furthermore, the phantom dimension is obviously invariant under Morita equivalences, since it only mentions compact objects, which are preserved by any equivalence of categories.

Note that if $E_{*}$ is countable, or, more generally, if $\mathcal{D}(E)$ is a Brown category, then phan. dim. $E \leqslant 1$ [Nee97, Chr98]. Very little else about the phantom dimension is known, except that it can be greater than one (as is shown in the above papers). The natural conjecture is that phan. dim. $H R$ should be the pure global dimension of $R$, but this is false for $R=\mathbb{Z} / 4$, whose pure global dimension is 0 whereas phan. $\operatorname{dim} . \mathbb{Z} / 4=1$. The problem here is the difference between finitely presented $R$-modules and compact objects of $\mathcal{D}(R)$.

In any case, we have the following proposition. 
Proposition 3.6. Suppose $E$ is an $S$-algebra. Then

r. gl. $\operatorname{dim} . E<$ (gh. dim. $E+1)$ (phan. dim. $E+1)$.

In particular, if $\mathcal{D}(E)$ is a Brown category, then

$$
\text { r. gl. } \operatorname{dim} . E \leqslant 2(\text { gh. } \operatorname{dim} . E+1) \text {. }
$$

This proposition is quite a bit weaker than the inequality for ordinary rings, mentioned above. This is because we cannot construct a resolution of an $E$-module in the same way as we can in algebra. But possibly this proposition can be improved.

Proof. Suppose gh. $\operatorname{dim} . E=n$ and phan. $\operatorname{dim} . E=k$. There is nothing to prove if either of these is infinite, so assume they are finite. Then any $(n+1)$-fold composite of ghosts is phantom, so any $(n+1)(k+1)$-fold composite of ghosts is a composite of $k+1$ phantom maps, and so is null.

\section{Gorenstein rings}

Recall that one of the themes of [HL09b] was that if $E$ is an $S$-algebra and r. gl. $\operatorname{dim} . E=0$, then there are very severe restrictions on $E_{*}$. In particular, we show that $E_{*}$ must be quasi-Frobenius, though much more is true. Since quasi-Frobenius rings are the same as 0-Gorenstein rings, a natural conjecture might be that if r. gl. dim. $E=n$, then $E_{*}$ is $n$-Gorenstein. Unfortunately, this is easily seen to be false, since $K O_{*}$ is not $n$-Gorenstein for any $n$, as we show in this section. However, we also show that if r.gl. $\operatorname{dim} . E=1$ and $E_{*}$ is a Noetherian domain, then $E_{*}$ is 1Gorenstein. We do not know if this statement is true for larger $n$, though it seems unlikely.

Recall that a (possibly noncommutative) ring $R$ is called Gorenstein if it is left and right Noetherian and $R$ has finite injective dimension as a left or right $R$-module. This generalizes the usual definition of Gorenstein in the commutative case, which is much used in algebraic geometry. If $R$ is Gorenstein, the right and left injective dimensions of $R$ must coincide, and if they are at most $n, R$ is called $n$-Gorenstein. These generalizations of quasi-Frobenius rings, which are just 0-Gorenstein rings, have been the object of much recent study. Chapter 9 of [EJ00] is a good place to start.

We begin by showing that $K O_{*}$ is not Gorenstein.

Proposition 4.1. The ring $K O_{*}$ is not $n$-Gorenstein for any $n$, though it is Noetherian and gl. $\operatorname{dim} . K O<\infty$.

Proof. If $K O_{*}$ were Gorenstein, it would be Cohen-Macaulay, which would mean that its depth would be equal to its Krull dimension. But

$$
K O_{*}=\mathbb{Z}_{(2)}\left[\eta, w, v, v^{-1}\right] /\left(\eta^{3}, 2 \eta, w \eta, w^{2}-4 v\right) .
$$

The only prime ideals in $K O_{*}$ are $(\eta)$ and the maximal ideal $(\eta, 2, w)$. So the Krull dimension is 1 . But there are no non-zero divisors in the maximal ideal, so the depth is 0 , and so $K O_{*}$ is not Cohen-Macaulay.

We now consider the case when gl. dim. $E=1$. 
Proposition 4.2. Suppose $E$ is an $S$-algebra with

$$
\text { r. gl. } \operatorname{dim} . E=\text { r. gl. dim. } E^{\mathrm{op}}=1 \text {, }
$$

and suppose that there are no nonzero maps from an injective (left or right) $E_{*}$ module to a projective (left or right) $E_{*}$-module. Then inj. dim. $E_{*} \leqslant 1$ as either a left or right $E_{*}$-module. Consequently, if $E_{*}$ is also left and right Noetherian, then $E_{*}$ is a 1-Gorenstein ring.

Proof. Let $R=E_{*}$. It suffices to show that inj. $\operatorname{dim} . R \leqslant 1$. We do this on just one side, since the hypotheses are left-right symmetric. For this, we embed $R$ into an injective module $I_{0}$, giving us the short exact sequence

$$
0 \rightarrow R \rightarrow I_{0} \rightarrow R_{1} \rightarrow 0 .
$$

We can realize this uniquely by an exact triangle in $\mathcal{D}(E)$

$$
E \rightarrow J_{0} \rightarrow M_{1} \stackrel{\delta_{0}}{\longrightarrow} \Sigma E
$$

in which $\delta_{0}$ is a ghost. We can then embed $\pi_{*} M_{1}$ into an injective module $I_{1}$, and get an analogous exact triangle

$$
M_{1} \rightarrow J_{1} \rightarrow M_{2} \stackrel{\delta_{1}}{\longrightarrow} \Sigma M_{1}
$$

in which $\delta_{1}$ is a ghost. Now the composite

$$
M_{2} \stackrel{\delta_{1}}{\rightarrow} \Sigma M_{1} \stackrel{\delta_{0}}{\rightarrow} \Sigma^{2} E
$$

is necessarily trivial, because r. gl. $\operatorname{dim} . E=1$. It is represented in the universal coefficient spectral sequence

$$
E_{2}^{s, t}=\operatorname{Ext}_{E_{*}}^{s, t}\left(\pi_{*} M_{2}, E_{*}\right) \Rightarrow \mathcal{D}(E)\left(M_{2}, E\right)_{t-s}
$$

on the 2-line by the extension

$$
0 \rightarrow R \rightarrow I_{0} \rightarrow I_{1} \rightarrow M_{2} \rightarrow 0
$$

which is trivial if and only if inj. $\operatorname{dim} . R \leqslant 1$. This element of $E_{2}$ is a permanent cycle, since it represents a map, but it must not survive the spectral sequence. Therefore, there must be a differential, necessarily a $d_{2}$, that hits it. The source of such a differential is a map $\pi_{*} M_{2} \rightarrow E_{*}$. But there are no nonzero maps like this, because $M_{2}$ is the quotient of an injective module. Thus, the extension above must be 0 , so inj. $\operatorname{dim} . R \leqslant 1$.

Now consider the case where $R$ is commutative Noetherian. Then every injective module is a direct sum of copies of the injective hulls $E(R / \mathfrak{p})$ of prime ideals $\mathfrak{p}$ (see $\left[\operatorname{Lam99}\right.$, Section 3I]). Every element of $E(R / \mathfrak{p})$ is killed by $\mathfrak{p}^{n}$ for some $n$. Thus, if $R$ is a domain, the only possible injective module that can map to a projective module is $E(R)$ itself. But $E(R)$ is divisible, so every element can be divided by every element of $R$. Thus as long as $R$ is a Noetherian domain that is not a field, there are no nonzero maps from an injective to a projective module.

We have therefore proved the following corollary.

Corollary 4.3. Suppose $E$ is an $S$-algebra with r.gl. dim. $E=1$, and $E_{*}$ is a commutative Noetherian domain that is not a field. Then $E_{*}$ is 1-Gorenstein. 


\section{References}

[ASS06] I. Assem, D. Simson, and A. Skowroński, Elements of the representation theory of associative algebras. Vol. 1, London Mathematical Society Student Texts, vol. 65, Cambridge University Press, Cambridge, 2006, Techniques of representation theory. MR 2197389 (2006j:16020)

[Beh06] M. Behrens, A modular description of the $K(2)$-local sphere at the prime 3, Topology 45 (2006), no. 2, 343-402. MR 2193339 (2006i:55016)

[BH84] K. A. Brown and C. R. Hajarnavis, Homologically homogeneous rings, Trans. Amer. Math. Soc. 281 (1984), no. 1, 197-208. MR 719665 (85e:16046)

[BKS04] D. Benson, H. Krause, and S. Schwede, Realizability of modules over Tate cohomology, Trans. Amer. Math. Soc. 356 (2004), no. 9, 36213668 (electronic). MR 2055748 (2005b:20102)

[Bou90] A. K. Bousfield, A classification of K-local spectra, J. Pure Appl. Algebra 66 (1990), no. 2, 121-163. MR 1075335 (92d:55003)

[Chr98] J. D. Christensen, Ideals in triangulated categories: phantoms, ghosts and skeleta, Adv. Math. 136 (1998), no. 2, 284-339. MR 1626856 (99g:18007)

[EJ00] E. E. Enochs and O. M. G. Jenda, Relative homological algebra, de Gruyter Expositions in Mathematics, vol. 30, Walter de Gruyter \& Co., Berlin, 2000. MR 2001h:16013

[EKMM97] A. D. Elmendorf, I. Kriz, M. A. Mandell, and J. P. May, Rings, modules, and algebras in stable homotopy theory, American Mathematical Society, Providence, RI, 1997, with an appendix by M. Cole. MR 97h:55006

[GH04] P. G. Goerss and M. J. Hopkins, Moduli spaces of commutative ring spectra, Structured ring spectra, London Math. Soc. Lecture Note Ser., vol. 315, Cambridge Univ. Press, Cambridge, 2004, 151-200. MR 2125040 (2006b:55010)

[Hil07] M. A. Hill, The 3-local tmf-homology of $B \Sigma_{3}$, Proc. Amer. Math. Soc. 135 (2007), no. 12, 4075-4086 (electronic). MR 2341960 (2008k:55008)

[HL09a] M. Hovey and K. Lockridge, The ghost dimension of a ring, Proc. Amer. Math. Soc. 137 (2009), no. 6, 1907-1913. MR 2480270

[HL09b] - Semisimple ring spectra, New York J. Math. 15 (2009), 219243. MR 2511136

[HL11] - The ghost and weak dimensions of rings and ring spectra, Israel J. Math. 182 (2011), 31-46.

[Hop02] M. J. Hopkins, Algebraic topology and modular forms, Proceedings of the International Congress of Mathematicians, Vol. I (Beijing, 2002), Higher Ed. Press, 2002, 291-317. MR 1989190 (2004g:11032)

[HPS97] M. Hovey, J. H. Palmieri, and N. P. Strickland, Axiomatic stable homotopy theory, Mem. Amer. Math. Soc. 128 (1997), no. 610, x+114. MR 1388895 (98a:55017) 
[HS99] M. Hovey and N. P. Strickland, Morava K-theories and localisation, Mem. Amer. Math. Soc. 139 (1999), no. 666, viii+100. MR 1601906 (99b:55017)

[Lam99] T. Y. Lam, Lectures on modules and rings, Graduate Texts in Mathematics, vol. 189, Springer-Verlag, New York, 1999. MR 1653294 (99i:16001)

[Nee97] A. Neeman, On a theorem of Brown and Adams, Topology 36 (1997), no. 3, 619-645. MR 1422428 (98e:18007)

[Sag08] S. Sagave, Universal Toda brackets of ring spectra, Trans. Amer. Math. Soc. 360 (2008), no. 5, 2767-2808. MR 2373333 (2008j:55009)

[Sch04] S. Schwede, Morita theory in abelian, derived and stable model categories, Structured ring spectra, London Math. Soc. Lecture Note Ser., vol. 315, Cambridge Univ. Press, Cambridge, 2004, 33-86. MR 2122154 (2005m:18015)

[Wol98] J. J. Wolbert, Classifying modules over K-theory spectra, J. Pure Appl. Algebra 124 (1998), no. 1-3, 289-323. MR 1600317 (99e:55013)

Mark Hovey hovey@member.ams.org

Department of Mathematics, Wesleyan University, Middletown, CT 06459, USA

Keir Lockridge klockrid@gettysburg.edu

Department of Mathematics, Gettysburg College, Gettysburg, PA 17325-1400, USA 